\title{
Detection Techniques for High Energy Neutrinos
}

\section{Giorgio Riccobene* Laboratori Nazionali del Sud - INFN}

E-mail: riccobenellns.infn.it

In the past decade the discovery and even identification of about hundred cosmic High Energy gamma-ray sources has pushed the field of astro-particle physics. Part of these sources are expected to be also accelerators of the observed Cosmic Rays and, thus, high energy neutrino sources. The observation of High Energy cosmic neutrinos, will definitely clarify the occurrence of hadronic acceleration in astrophysical objects and eventually identify sources of Cosmic Rays. Theoretical estimates indicate that a detection area of the order of a few $\mathrm{km}^{2}$ is required to measure the HE cosmic $v$ fluxes. The underwater/ice optical Čerenkov technique is widely considered the most promising experimental approach to build high energy neutrino detectors in the TeV-PeV energy range. After the first generation of underwater/ice neutrino telescopes (Baikal, AMANDA and ANTARES), the quest for the construction of $\mathrm{km}^{2}$ size detectors have already started. At the South Pole the construction of the IceCube neutrino telescope is in an advanced stage, while the ANTARES, NEMO and NESTOR collaborations together with several other European Institutions take part to KM3NeT aiming at the installation of $\mathrm{a} \mathrm{km}^{3}$-scale neutrino telescope in the Mediterranean Sea. The detection of fainter $>\mathrm{PeV} v$ fluxes, will require the construction of even larger detectors ( $>>1$ GTon scale), using novel detection techniques such as the Radio-Cherenkov and acoustic detection. ANITA, a balloon based, detector has already provided strong limits to the cosmic neutrino fluxes above $100 \mathrm{PeV}$ and the construction of IceRay, a very large detector based on the radio-acoustic technique complementing the IceCube detector, has been proposed. An intense R\&D activities is also ongoing aimed at the construction of neutrino acoustic detectors underwater.

XLVIII International Winter Meeting on Nuclear Physics, BORMIO2010

January 25-29, 2010

Bormio, Italy

\footnotetext{
*Speaker.
} 


\section{Introduction}

The main messengers of the High Energy (HE) Universe are, nowadays, hadrons (protons and heavier nuclei) and gamma rays, but the future detection of neutrinos and gravitational waves will be fundamental. Each of these probes reveals, in fact, peculiar behaviours of cosmic sources, thus only a multi-messenger astronomy, i.e. based on contemporary observation of astrophysical objects with different particle detectors, will allow to get a deeper insight on the HE Universe and on the mechanisms responsible for the production of high energy particles in astrophysical sources. The most appealing feature of neutrinos -chargeless particles interacting only weakly with matter- is that they can travel through the Universe without being deflected or absorbed. In fact, the horizon for $E>10^{12} \mathrm{eV}$ photons is limited to few tens $\mathrm{Mpc}$, by the interaction on the diffuse cosmic microwave and infrared background: $\gamma_{H E}+\gamma_{C M B, I R} \rightarrow e^{+} e^{-}$; while extremely protons of $E>10^{19.5}$ $\mathrm{eV}$, loose energy within few tens of Mpc by their interaction with the Cosmic Microwave Background Radiation: $p+\gamma_{C M B, I R} \rightarrow \Delta^{+}$, named GZK effect, after the scientists Greisen, Zatsepin and Kuzmin [1],[2]. Therefore the detection of HE neutrinos $\left(E_{v}>1 \mathrm{TeV}\right)$ should allow us to extend our knowledge to the far high energy Universe and to probe the dense core of the most powerful cosmic accelerators. The price to pay is that neutrinos are extremely difficult to detect and therefore the opening of high neutrino astronomy requires huge detectors. Theoretical expectations on cosmic high energy neutrino fluxes vary a lot according to different models, however it is expected that detectors of masses of few GTons will allow the identification of TeV-PeV neutrino fluxes; hundred GTon-scale detectors will be able to measure neutrino fluxes at $E_{v}>10^{18} \mathrm{eV}$. The use of natural media as $v$ target is therefore mandatory to build such detectors with affordable budget.

\section{The cosmic rays, gammas and neutrinos connection}

Although the first studies on Cosmic radiation date back to the beginning of XX century [3], Cosmic Rays (CR) are still a puzzling subject for physicists. Present data show that the CR flux extends over 10 orders of magnitude in energy, up to $3 \cdot 10^{20} \mathrm{eV}$, with a power law spectrum, showing an almost constant $\alpha=-2.7$ spectral index [5]. The measured composition of the bulk of $\mathrm{CR}$ is hadron dominated, with about $87 \%$ protons, $9 \%$ alpha particles and the rest shared among heavier nuclei, photons and electrons [4]. In spite of the fact that CR spectrum has been measured with great accuracy up to $10^{20} \mathrm{eV}$, a conclusive evidence of connection with sources is still missing. The arrival direction at the Earth of cosmic rays (up to $10^{18} \mathrm{eV}$ ) is, in fact, randomized by the Galactic ( $B \simeq 3 \mu \mathrm{G})$ and intergalactic $(B \simeq \mathrm{nG})$ magnetic fields. In figure 1 (left-hand side) the CR spectrum is multiplied by $E^{2.7}$, revealing the presence of different trends with increasing energy. At $E<10^{14.5} \mathrm{eV}$ the $\mathrm{CR}$ spectral index is $\alpha \simeq 2.7$, between $E \simeq 10^{14.5} \mathrm{eV}$ and $E \simeq 10^{17.5}$ $\mathrm{eV}$ the spectral index becomes softer $\left(\alpha \simeq 3\right.$ ), above $E=10^{18.5}$ the spectral index changes again to $\alpha \simeq 2.7$. The two breaks in the energy spectrum are usually referred as knee and ankle respectively. Above $E \simeq 10^{19} \mathrm{eV}$, the CR flux measured at the Earth is as low as 1 particle/( $\mathrm{km}^{2}$ year), and above $10^{20} \mathrm{eV}$ the observed CR spectrum is suppressed.

The bulk of CR spectrum is understood in terms of particles accelerated in astrophysical sources through the Fermi-Bell statistical acceleration mechanism [6], [7]). In explosive astrophysical sources (such as Supernova Remnants -SNR-, Active Galactic Nuclei-AGN-, Gamma 

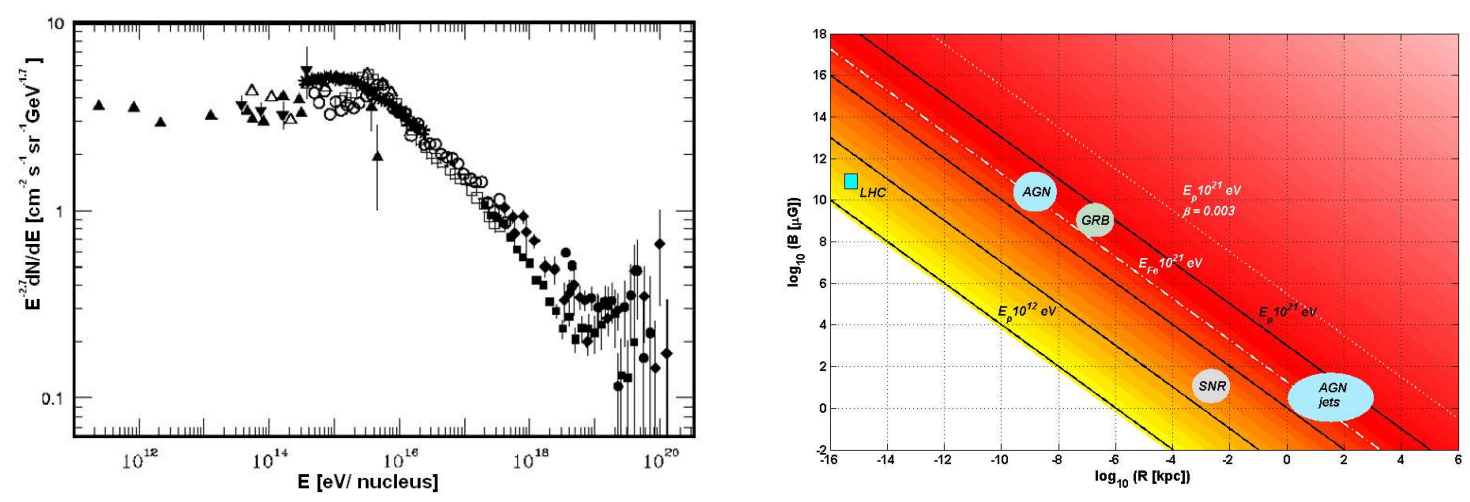

Figure 1: Left: The measured CR spectrum multiplied by $E^{2.7}$; two main changes of the spectral index occur at $E \simeq 10^{14.5} \mathrm{eV}$ (knee) and at $E \simeq 10^{14.5} \mathrm{eV}$ (ankle), see text. Right: The Hillas Plot. Astrophysical sources accelerate high energy protons and nuclei through diffuse shock acceleration. The particle maximum energy is a function of the source dimensions and magnetic field.

Ray Bursters -GRB-) a plasma of charged particles $\left(e^{+} e^{-}\right.$or/and $\left.p^{+} e^{-}\right)$can be driven by strong shock waves and contained by magnetic fields. Charged particles gain energy, statistically, crossing the shock front from the downstream to the upstream region and viceversa. Since the particle confinement is a function of the object dimensions $(R)$ and strength of the local magnetic field $(B)$, Hillas [8] provided an useful plot (see Fig. 1, right-hand side) to determine the maximum energy that a particle can reach in a given astrophysical source.

From many indications, Galactic SNRs are considered the most probable sources of the CR flux below the ankle. In this energy region, the sky-map of observed CR is almost isotropic, due to randomization of charged particle arrival direction in the Galactic magnetic field. Above the knee energy, the total CR flux decreases and the composition increases in metallicity, as expected in the scenario of SNR acceleration: protons are not confined any more in the Galaxy, and only nuclei with higher $Z$ (synthesized in the nuclei fusion chain of SNs) remain trapped in the Galaxy. in this scenario, protons accelerated in SNR via the Fermi-Bell mechanism, may interact with ambient radiation and/or matter, within the source or with nearby gas clouds producing pions. Neutral pions then decay into a pair of $\gamma_{\mathrm{s}}$, while charged pions decay producing neutrinos. The expected "hadronic" gamma flux (produced in the $\pi^{0} \rightarrow \gamma \gamma$ channel) therefore follows primary proton flux. The observation by the HESS telescope of TeV gamma rays emitted by the ROSAT and ASCA X-Ray source RXJ1713.7-3946 [9], indicated, for the first time, features suggesting the presence of proton Fermi acceleration. Similarly a muon neutrino flux is expected to be produced and, if muons do not loose a large fraction of energy before decay, high energy electron neutrinos are also produced. Taking into account neutrino oscillations during the source-Earth journey, equipartition between the three leptonic flavors is expected at the Earth. Above $E>10^{18.5} \mathrm{eV}$ (the ankle region) the CR spectrum is similar to the pre-knee region: the spectral index is close to 2.7 and the flux appears to be proton-dominated, though new experimental data from the Pierre Auger Observatory seem to favor a higher $Z$ composition [10]. Since the known Galactic sources (SNR, Microquasars, Pulsar Wind Nebulae) cannot accelerate particles to extremely high energies, as shown in figure 1, the detection of cosmic protons with energies up to $E>10^{19} \mathrm{eV}$ suggests the presence of powerful 
extragalactic accelerators, such as AGN and GRB. In these object also the production of $E>\mathrm{TeV}$ gamma and neutrino fluxes is expected, by interaction of Fermi protons with the ambient photon field (mainly). As shown before, above $10^{20} \mathrm{eV}$ the CR flux is suppressed, as expected by the GZK effect [1],[2]. Since AGNs and GRBs lie at cosmological distances, protons and nuclei accelerated in these sources loose energy before reaching the Earth by interaction with the Cosmic Microwave Background Radiation; HE gamma rays are absorbed as well. Neutrino, therefore, are promising probes to investigate violent and far astrophysical sources in the Universe.

\section{High energy neutrino detection}

In order to identify astrophysical sources, neutrino detectors must reconstruct both the detected neutrino energy and direction, thus they are commonly referred as Neutrino Telescopes. At energies above few hundreds $\mathrm{GeV}$, neutrinos are detected through deep-inelastic scattering of the $v$ with a target nucleon $N$. In the $v+N \rightarrow l+X$ interaction, the lepton $l$ escapes while the hadronic debris $X$ leads to a hadronic cascade. The initial neutrino energy $E_{v}$, is shared among the lepton $E_{l}$ and the hadronic cascade. The cascade carries $E_{h}=y \cdot E_{v}$, where $y$ is the Bjorken inelasticity parameter, with a mean value of $\langle y\rangle \simeq 0.25$ at very high energies, and a very broad distribution; the lepton takes the remaining energy [11]. In weak charged-current (CC) interactions the outgoing lepton is charged and it preserves the neutrino flavour $(e, \mu$ or $\tau)$. In neutral-current (NC) interactions, the outgoing lepton is a neutrino, thus only the hadronic cascade is detectable. The detection of the $v$ interaction is, therefore, based on the observation of the outgoing charged lepton and/or of the hadronic cascade. The $v N$ cross section is as low as $\sigma_{v N} \simeq 10^{-35} \mathrm{~cm}^{2}$, at $\simeq 1 \mathrm{TeV}$, increasing linearly with the neutrino energy up to $5 \mathrm{TeV}$ energy, above this value its slope changes to $E^{-0.4}$ [12]. The increase of the neutrino cross section as a function of energy implies also that at $E>$ $10 \mathrm{TeV}$, the Earth is not transparent to neutrinos. Due to the low $v N$ cross section and to the faint expected astrophysical $v$ fluxes $\left(\propto E_{v}^{-2}\right)$, the detectors must have a $v$ interaction target mass of several GTons for $E_{v} \simeq 10^{12} \div 10^{17} \mathrm{eV}$ and much larger for higher energies. For this reasons the use of natural media was suggested to detect cosmic neutrinos. Depending on the candidate interaction target medium and on the energy range to explore, different experimental techniques were proposed.

\section{Underwater/ice Čerenkov detectors}

The underwater/ice Čerenkov technique is, at present, the most promising and advanced. The idea, suggested by Markov [13], is based on instrumentation of large volumes of sea/lake water or polar ice, in order to detect the charged leptons (in particular muons, as we will discuss) emerging from a CC neutrino interaction. Underwater(ice) Čerenkov neutrino detectors are large arrays of optical sensors, typically photomultiplier tubes (PMTs) of about 10" diameter, which permit charged leptons tracking, by timing the Čerenkov light wavefront radiated by these particle. In water and ice, relativistic particles radiate Čerenkov light mainly in the UV-blue wavelengths. In both media the refractive index in this spectral region is $n \simeq 1.35$, and photons are emitted the along particle track at the angle $\vartheta \simeq 42^{\circ}$ and symmetrically in $\varphi$. The time sequence of Čerenkov photons hits on PMTs is correlated in space and time, permitting the reconstruction of the Čerenkov 
wavefront, therefore of the particle track, from the experimental data. The reconstructed track direction is however affected by experimental indetermination: the error on PMTs position and on absolute photon hit time, due to photon scattering in the medium, PMT transit time spread and to detector time calibration. Photon scattering in the medium is an important issue, since scattering deflects Čerenkov photons, affecting track direction reconstruction. In ice the scattering length of light is only few tens of $\mathrm{cm}$, in water it is about $100 \mathrm{~m} \mathrm{[14].} \mathrm{It} \mathrm{is} \mathrm{worth} \mathrm{to} \mathrm{mention} \mathrm{that} \mathrm{particle}$ energy loss via Čerenkov radiation is only a small fraction of the total one, and the number of Čerenkov photons (UV-blue) is only 300 per $\mathrm{cm}$ of track. Given this small amount of light, photons hits PMTs only if the average distance between optical sensors is not larger than the light absorption length in the medium. The medium optical properties, thus, determine the detector granularity (i.e. the PMT density) and its size. Water is transparent only to a narrow range of wavelengths (350 $\mathrm{nm} \leq \lambda \leq 550 \mathrm{~nm})$. In particular, for deep polar ice $L_{a}(\mathrm{UV}$-blue $\mathrm{nm}) \simeq 100 \mathrm{~m}$ [15], and it is about $70 \mathrm{~m}$ for clear ocean waters [14]. This leads to the use of not less than $\simeq 5000$ optical sensors per $\mathrm{km}^{3}$. The "golden channel" for astrophysical neutrino detection is the $v_{\mu} \mathrm{CC}$ interaction. The muon range in water is, at $E \simeq \mathrm{TeV}$, of the order of kilometres, therefore the $v_{\mu}$ interaction can take place either within the detector or far outside it, providing a flux of high energy muons, either contained or crossing the detector. The muon direction is recovered from the reconstruction of the Čerenkov wavefront, radiated along the muon track, within the detector instrumented volume. The detection of the neutrino-induced muon also allows "neutrino astronomy": the angle between the outgoing muon and the interacting neutrino decreases as a function of neutrino energy: at $E_{v}>1$ $\mathrm{TeV}$, the muon track is almost co-linear to the $v_{\mu}$ one and permit pointing back to the $v$ cosmic source. These detectors are, in fact, also named as Neutrino Telescopes. For the muon neutrino detection, up-going or horizontal muon tracks are preferred. In fact, when an upward going muon is reconstructed this is a unique signature of a neutrino event, being the up-going atmospheric muon background completely filtered out within few tens of $\mathrm{km}$ of water. The suppression of the intense down-going atmospheric muon flux is achieved installing the detector at large water(ice) depth: the muon stopping power of $3000 \mathrm{~m}$ of water is equivalent to the one of $1 \mathrm{~km}$ of rock. Neutrino telescopes are also expected to disentangle between neutrino flavours by reconstructing the Čerenkov wavefront shape of the event which depends on the different propagation of $e, \mu$ and $\tau$ in water (and ice). In case of $v_{e} \mathrm{CC}$ interactions, the final state involves high energy electrons that provide a high energy electromagnetic shower superimposed on the hadronic one. Both showers extend for few tens of metres from the $v$ interaction point, thus only interactions that are fully contained into the detector instrumented volume, or very close to it, can be identified. At distance of few hundreds metres from the shower, the shape of the light wavefront is similar to an expanding sphere, thus, the neutrino direction is difficult to reconstruct. On the other hand, showers involve a large number of charged particles $\left(N \propto E_{v}\right)$ radiating Čerenkov light and, in this case, the lepton energy can be well estimated from the shower light yield. Photon yield is also effectively used to estimate energy in UHE $v_{\mu}$ detection, for which the muon generates high energy showers along its path. The scenario depicted for $v_{e}$ is similar to the case of tau neutrino detection: up to $E_{v} \simeq 1 \mathrm{PeV}$ the $\tau$ decay length is too short $\left(\simeq 50 / E_{\tau} \mathrm{mm} / \mathrm{TeV}\right)$ to reconstruct the tau decay shower from the hadronic one. When the $\tau$ path is about $100 \mathrm{~m}$ long, then, the two cascades can be separated and the event topology shows the typical signature of a "double light bang".

Based on present astrophysical models and detailed Monte Carlo simulations, a rate of about 
70 up-going events per year is expected in an underwater/ice detector of $1 \mathrm{~km}^{3}$ volume equipped with about 5000 optical sensors (for $E_{\mu} \simeq 1 \mathrm{TeV}$ threshold). The expected angular resolution is of $\simeq 0.1^{\circ}$ for $E_{\mu}>10 \mathrm{TeV}$ [16].

A number of demonstrator detectors have been built in the last decade to cope with major technological issues due to detector installation at high pressure, low temperature (polar ice), salinity(deep sea). To date, the most advanced HE neutrino detector is IceCube, born from the experience of AMANDA, and installed in the Geographical South Pole. The detector will consists of 80 vertical strings (to date 73 strings are deployed), each equipped with 60 Digital Optical Modules (DOM), deployed between $1450 \mathrm{~m}$ and $2450 \mathrm{~m}$ depth below the surface, as shown in figure 2 (left). The DOM spacing along the string is $17 \mathrm{~m}$ and the strings are placed on a hexagonal lay-out with $125 \mathrm{~m}$ spacing, obtaining $1 \mathrm{~km}^{3}$ of instrumented volume. The DOM contains a 10" PMT and digital read-out electronics to permit signal waveform analysis, leading to a relevant improvement of detector performance compared to AMANDA, that used analogue PMT read-out. An improvement of the telescope sensitivity in the low energy range has been recently achieved thanks the addition of six more densely instrumented strings, deployed in the bottom center of the telescope to form the so-called DeepCore detector. DeepCore will lower the IceCube threshold for muons to about $10 \mathrm{GeV}$, thus allowing to address more effectively low energy physics issues and especially to increase the sensitivity for the indirect search of Dark Matter [17]. In the IC40 configuration (IC40, 365 days livetime from 2008 to 2009) IceCube has set an upper limit to diffuse neutrino flux, for a $E_{v_{\mu}}^{-2}$ spectrum, of $1.7 \cdot 10^{-8} \mathrm{GeV}$, that does not show any excess with respect to the expected atmospheric neutrino flux, within systematics errors [18]. The good detector angular resolution (about 1 degree) allowed also a detailed study of the neutrino event direction. The $v$ sky map seen by IceCube including both up-going and down-going neutrinos is reported in figure 2 (right). Down-going events are selected using an energy threshold $\left(E_{\mu} \geq 10^{5} \mathrm{GeV}\right)$ sufficiently high to get rid of atmospheric muons. No significant excess from any sky direction has been observed up to now. In figure 3 the sensitivity to point-like neutrino sources, as a function of source declination, is reported [19].
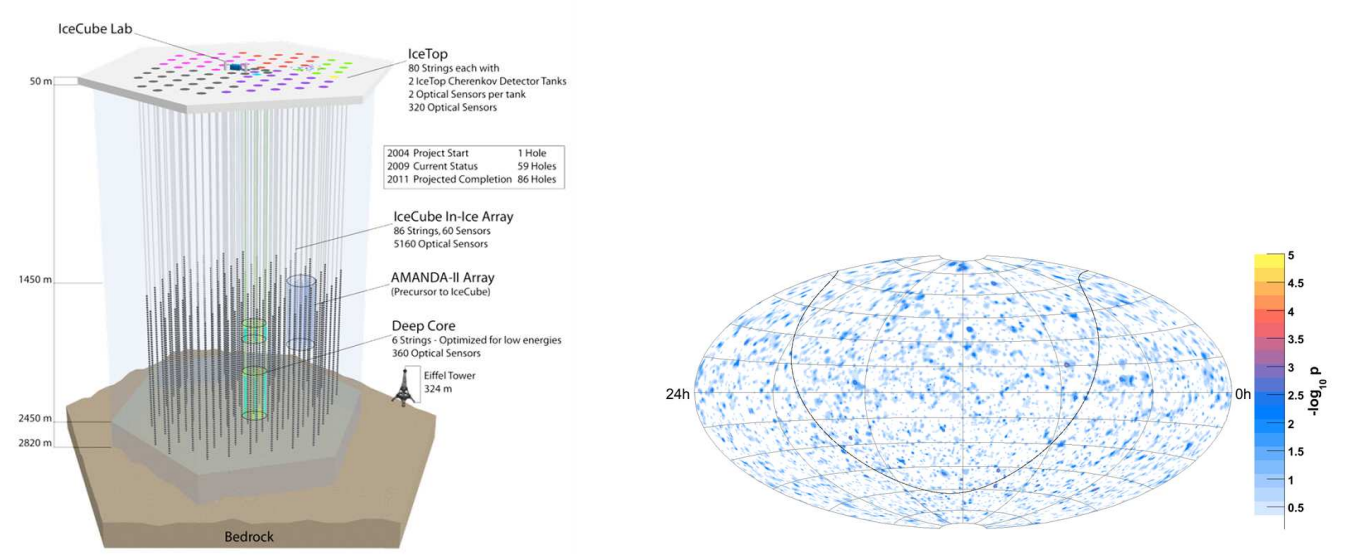

Figure 2: Left: Pictorial view of the IceCube detector. Right:The neutrino sky map seen by Icecube.

The pioneer effort to build a large neutrino telescope in the Northern Hemisphere, was conducted by the Baikal Collaboration, based in Lake Baikal [20]. Due to limited water depth $(<1500$ $\mathrm{m})$ and not optimal optical properties of lake-waters, the present detector can be hardly expanded 
to reach the required angular accuracy and detection volume. From the '90s three collaborations are also active to build a $\mathrm{km}^{3}$ neutrino telescope in the Mediterranean Sea: ANTARES, NEMO and NESTOR. ANTARES is presently the largest neutrino telescope operating in the Northern Hemisphere [21]. The detector, deployed at a depth of about $2400 \mathrm{~m}$, offshore Toulon (France) is made of 12 strings, hosting 75 Optical modules each. The present detector size is about $0.035 \mathrm{~km}^{3}$. Thanks to its high PMT granularity, the ANTARES telescope has an energy neutrino threshold of about $20 \mathrm{GeV}$ for reconstructed muon events and detector performance dramatically improves with increasing energy reaching at neutrino energy of $10 \mathrm{TeV}$ an angular resolution of 0.3 degrees. In its final configuration the detector has well measured the atmospheric muon flux as a function of slant water depth indicating good agreement between ANTARES data, other measurements and expectations. The search for cosmic neutrino point-like sources was also performed. Although no significant excess was found, the present detector sensitivity represents the best existing limits for point-like neutrino sources in the Southern Sky, even if compared to the multi-year experiment SuperKamiokande [22]. The NEMO (NEutrino Mediterranean Observatory) Collaboration has conducted an intense $\mathrm{R} \& \mathrm{D}$ programme aimed at developing and validating key technologies for a cubic-kilometre scale underwater neutrino telescope [23]. Moreover, after more than 30 sea campaigns an optimal deep sea site for the installation of the $\mathrm{km}^{3}$-scale detectors was found in the Mediterranean Sea offshore the village of Capo Passero. The site located at a depth of $3500 \mathrm{~m}$ and $90 \mathrm{~km}$ off-shore the Southern Sicily coast, shows optimal features to host the $\mathrm{km}^{3}$ detector: low optical background ( $30 \mathrm{kHz}$ on 10" PMTs at 0.5 s.p.e. threshold), blue light absorption length of $70 \mathrm{~m}$ (close to optically pure water), low currents $(3 \mathrm{~cm} / \mathrm{s}$ in average) and low sedimentation rate. The site is located on a wide abyssal plateau, showing very stable environmental conditions and a flat seabed morphology over allowing for possible extension of the telescope. NEMO has also successfully proven a new mechanical structure, a semirigid tower, that can be a favourable solution for deep sea detectors, compared to the string. In this structure several Optical Modules are displaced in a 3D geometry increasing the muon track reconstruction capabilities of a single structure compared to 1D line structures. A small-size tower prototype was tested offshore Catania (Sicily) in 2006, demonstrating excellent capabilities in measuring the atmospheric muon flux [24]. The efforts of ANTARES, NEMO and NESTOR are now converging in the KM3NeT Consortium, funded by the EU aims at the construction of a cubic-kilometre-scale neutrino telescope in the the Mediterranean Sea [16]. Due to the Earth rotation, the telescope proposed by KM3NeT will allow to see up-going neutrinos from about $3.5 \pi$ and to survey of a large part of the Galactic Plane, including the Galactic Centre and many intense $\mathrm{TeV}$ gamma sources, obscured to IceCube. The goal of the project is to achieve a sensitivity for point-like sources of about $1 \cdot E_{v}^{2} \Phi_{v} \simeq 10^{-9} \mathrm{GeV} \mathrm{cm}^{-2}$ $\mathrm{s}^{-1}$ in 1 year [16]. This result is expected to be reached thanks to larger detector size compared to IceCube and thanks to better angular resolution (see figure 3) [25].

\section{Ultra High Energy neutrino detection}

At energies higher than $10 \mathrm{PeV}$ the expected neutrino flux is so low that $\mathrm{km}^{3}$-scale detectors based on optical Čerenkov detection are too small to detect UHE neutrino events. For this reason different, complementary techniques have been investigated with the aim of observing extremely high energy neutrino events, e.g. neutrinos produced in GZK interactions. These techniques rely 


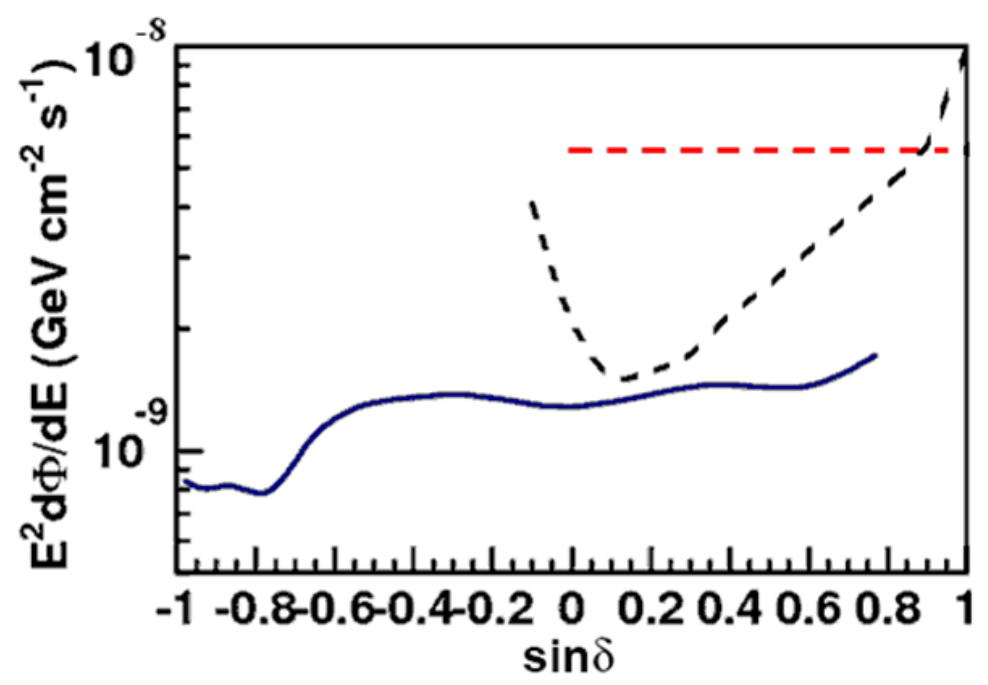

Figure 3: Expected sensitivity to $E^{-2}$ neutrino point sources as a function of source declination for IceCube (red line: standard analysis [26]; black line: improved analysis [19]) and KM3NeT (blue line from [25]).

on the identification of a UHE neutrino interaction through the detection of coherent radiation produced by neutrino-induced cascades - that propagates in dense media for very large distances. Hadronic showers, produced at the vertex of the UHE neutrino interaction, or electromagnetic showers, produced by $e$ outgoing a $v_{e}$ interaction, radiate coherent radio and acoustic emissions. Radio waves have typical attenuation lengths of few $\mathrm{km}$ in the ice and the attenuation length for acoustic waves in the sea is also of the order of several $\mathrm{km}$. Therefore, a sparse array of acoustic or radio sensors can be used to reconstruct the UHE $v$ interaction vertex. Askaryan [27] first suggested that natural media such as the polar ice-cap, salt domes and the lunar regolith could provide huge target material for neutrino interaction, being at the same time optimal transparent radiators for electromagnetic Radio Frequency (RF) signals. In such media, particle cascades induced by ultra high energy neutrinos are very compact, consisting of a bunch of relativistic charged particles displaced in a volume of a few $\mathrm{dm}^{3}$, which develops at the speed of light over a distance of few tens meters from the vertex of the neutrino interaction, before dissipating its energy into residual ionization in the medium. The average number of electrons and positrons near total shower maximum is of order the cascade energy, $E_{c}$, expressed in $\mathrm{GeV}: N^{e^{+} e^{-}}=E_{c} / 10^{9}$. During the e.m. cascade development a negative charge accumulates due to Compton scattering of photons and to annihilation of positrons interacting with the medium's electrons. The charge asymmetry is about $20 \%$ at the shower maximum. The relativistic motion of this negative charge, distributed in a volume of few $\mathrm{cm}^{3}$, in the medium produces coherent Čerenkov radiation in the $\mathrm{RF}$ range $f \simeq 100 \mathrm{MHz}$ $\div 1 \mathrm{GHz}$. A set of experiments at the Stanford Linear Accelerator center have now clearly confirmed the effect and described the radio signals shape, waveform, and amplitude versus deposited energy relation [28]. In Antarctic ice, where the refraction index at these frequencies is $n \simeq 1.8$, the Čerenkov angle is is at about 56 degrees, with a few degree spread along the shower track. Different experiments are now pursuing the measurement of the GZK neutrino fluxes through the Askaryan radio technique. The most stringent limit to ultra High Energy neutrino fluxes were set by ANITA that searched for neutrino-induced radio pulses emerging from the polar ice-cap. The 
ANITA detector was operated during 3 balloon flights above the South Pole. The integral sensitivity, calculated on a pure $E^{-2}$ spectrum for the energy range $10^{18.5} \mathrm{eV} \div 10^{23.5} \mathrm{eV}$ was evaluated to be $E_{v}^{2} \Phi_{v}=2 \cdot 10^{-7} \mathrm{GeV} \mathrm{cm}^{-2} \mathrm{~s}^{-1} \mathrm{sr}^{-1}$ [29].

Another technique for EHE $v$ detection is based on the identification of the acoustic signature of neutrino induced showers in water. Though the studies on this technique are still in an early stage, its potential use to build very large neutrino detectors water is appealing, thanks to the optimal properties of water as sound propagator [30]. The idea of acoustic neutrino detection, first proposed by Askaryan in 1957 [31], is based on the reconstruction of hadronic and electromagnetic showers produced by neutrino interaction in dense media. As discussed above, both in the case of $\mathrm{CC}$ or NC interaction, the hadronic cascade carries about $25 \%$ of the neutrino's energy. In case of CC interaction of a $v_{e}$, an e.m. shower is also produced close to the neutrino interaction vertex. The deposit of the cascade energy in a small volume of water, cylindrical in shape with a length $L_{c}$ of few tens metres and a few centimetres in radius $r_{c}$ turns out in heating of the medium, therefore in its expansion. The typical time of the energy deposition along the shower axis, almost co-linear with the neutrino direction, is of the order of $L_{C} / c \simeq 10^{-7} \mathrm{~s}$, while the expansion time is of the order of $r_{c} / c_{\text {sound }} \simeq 10^{-4} \mathrm{~s}$. The net result is the coherent production of a mechanical wave, "pancake-shaped" propagating, in a homogeneous medium, perpendicularly to the shower axis. Acoustic pulses from particle showers were first observed at Brookaven NL in 1979, using a beam of $200 \mathrm{MeV}$ protons with a total energy deposit in water of about $10^{18} \mathrm{eV}$. According to Learned's theoretical work [32], the acoustic pulse is bipolar, following the second time derivative of the temperature of the excited medium. The frequency spectrum of the signal is a function of the transverse spread ( $r_{\text {cascade }}$ ) of the shower, with typical maximum amplitude in the range of few tens $\mathrm{kHz}$. The amplitude of the bipolar signal is proportional to the deposited energy and to the medium properties: the thermal expansion coefficient $(\beta)$, the sound velocity $\left(c_{s}\right)$ and the specific heat capacity $\left(C_{p}\right)$. A rule of thumb to calculate the acoustic pulse amplitude at 1 metre produced by a neutrino of energy $E_{v}$, impinging in a dense medium is

$$
P_{0} \simeq 0.25 E_{v} \cdot \Gamma E_{v} / V_{c} \simeq 2 \Gamma E_{v} \cdot 10^{-19}[\mathrm{~Pa} / \mathrm{eV}]
$$

where $V_{c}$ is the volume of the medium where the shower deposits its energy, the factor 0.25 is the fraction of $E_{v}$ (in units of $[\mathrm{eV}]$ ) transferred to the shower and $\Gamma=c_{s}^{2} \beta / C_{p}$ is the (dimensionless) Gruneisen coefficient of the medium [33] . The $\Gamma$ coefficient for deep Mediterranean Sea water is about 0.12 , about a factor ten larger in polar ice and about 3.2 in compact mineral salt. Since, at frequencies of few tens $\mathrm{kHz}$, acoustic pulses can travel large distances in ice, seawater and salt, these natural media can be used to build large volume neutrino detectors instrumented with sparsely spaced arrays of acoustic sensors. Assuming radial propagation of the sound wave and that the sound absorption length in water is $L_{a}^{\text {sound }} \simeq 10 \mathrm{~km}$ at $20 \mathrm{kHz}$, the pulse amplitude produced by a $E_{v}=10^{20} \mathrm{eV}$ neutrino, recorded at $1 \mathrm{~km}$ distance, is expected to be about $15 \mathrm{mPa}$. Ice, despite the Gruneisen coefficient is larger, suffer for a stronger sound absorption ( $L_{a}^{\text {sound }} \simeq 300 \mathrm{~m}$, at about $400 \mathrm{~m}$ depth) compared to water. The energy threshold for neutrino acoustic detection is set by the ratio between ambient noise and signal. In the frequency range of interest for neutrino detection $(10 \div 40 \mathrm{kHz})$, the acoustic ambient noise amplitude in deep sea adds up about few $\mathrm{mPa}$. This permits, in a first approximation, the discrimination of acoustic signals originated by 
neutrinos having $E_{V}>10^{19} \mathrm{eV}$ (assuming $1 \mathrm{~km}$ distance). The possibility to use time and direction coincidence can help to reduce the abient noise by at least one order of magnitude.

On the experimental side, in recent, years the possibility of using hydrophones installed on military array and the infrastructures of new underwater/ice Čerenkov telescopes, has permitted to several experimental groups to start R\&D activities on acoustic detection. The SPATS team installed four acoustic test lines, each equipped with 7 transmitters and 7 receivers, within the IceCube detector. SPATS permitted the first studies of deep ice acoustic properties, using calibrated pingers deployed at several depths and distances. Present results show that sound attenuation length in ice is about $300 \mathrm{~m}$, a value much less than expected from theoretical estimates [34]. In the framework of the activities of the ANTARES, NEMO and KM3NeT, two small size experiments were run in order to test acoustic devices, measure acoustic noise in deep sea and test "neutrino-like" acoustic event detection. AMADEUS, a system of 24 hydrophones was deployed using the ANTARES infrastructure [35], and NEMO-OnDE, deployed at offshore Catania. The NEMO Collaboration is also designing an innovative acoustic position system for the $\mathrm{km}^{3}$ detector that will be installed on a KM3NeT prototype to be deployed in Capo Passero in 2011. The system, an array about 30 hydrophones, will be able to work both as positioning system for the Č detector and autonomous acoustic detector [36].

\section{Conclusions}

High energy neutrino telescopes are expected to shed new light on the High Energy Universe [37]. These detectors may discover the occurrence of hadronic processes in astrophysical sources and eventually detect sources of Cosmic Rays. Perspectives for HE neutrino detection have dramatically improved in the last decade due to the huge technological progresses. At South Pole, IceCube, will soon reach the sensitivity to detect first astrophysical neutrino sources. On the other hand, after many years prototyping and validation of deep sea technologies, the KM3NeT consortium is ready for the start-up of the construction phase of the $\mathrm{km}^{3}$ scale underwater neutrino telescope in the Mediterranean.

A great effort is also addressed to the detection of UHE neutrinos: the sensitivity reached by ANITA already allowed to exclude the exotic cosmological models, that foreseen the production of ultra high energy neutrino fluxes from annihilation of heavy Dark Matter. Moreover, after the results of several pioneering experiments, the construction of very large array based on radio Čerenkov techniques, and acoustic detection, could be affordable in the future years.

\section{References}

[1] Greisen K. et al., Phys. Rev. Lett., 16, 748 (1966);

[2] Zatsepin G. T. and Kuzmin V. A., JETP Lett., 4, 78 (1966);

[3] Hess V., Phys. Z., 13, 1084 (1913);

[4] Gaisser T. K., Cosmic Rays and Particle Physics, Cambrige University Press, Cambridge (1990);

[5] Cronin J. W., Gaisser T. K. and Swordy S. P., Cosmic Rays at the Energy Frontier, Scientific American (1997); 
[6] Fermi E., Physics Review, 75, 1169 (1949);

[7] Bell A. R., MNRAS, 182, 147 (1978);

[8] Hillas A. M., Annu. Rev. Astron. Astrophys., 22, 425 (1984);

[9] Aharonian. F. et al., Nature, 432, 75 (2000);

[10] Abbasi R. et al., Proceedings of the 31th Int. Conf. on Cosmic Rays, Lodz - Poland 2009, arXiv:0906.2319 (2009);

[11] Okun L., Leptons and quarks, North Holland, Amsterdam (1982);

[12] Gandhi R. et al., Physical Review, D58, 93009 (1998);

[13] Markov M. A. and Zheleznykh I. M, Nuclear Physics, 27, 385 (1961);

[14] Mobley C. D., Light and Water: radiative transfer in natural waters, Academic Press, San Diego (1994);

[15] Ackermann M. et al., J. Geophys. Res., 111, D13203 (2006);

[16] Bagley P. et al., Conceptual Design Report for a Deep-Sea Research Infrastructure Incorporating a Very Large Volume Neutrino Telescope in the Mediterranean Sea (2008). Availbale at http://www.km3net.org/CDR/CDR-KM3NeT.pdf ;

[17] Wiebusch C. for the IceCube Collaboration, Proceedings of the 31th Int. Conf. on Cosmic Rays, Lodz - Poland(2009);

[18] DeYoung T. for the IceCube Collaboration, Modern Physics Lett., A24, 1543 (2009);

[19] Abbasi R. for the IceCube Collaboration, Astrophysical Journal Letters, 701, L47 (2009);

[20] Ayutdinov V. for the Baikal Collaboration, Nucl. Instr. and Meth., A602,14 (2009);

[21] Montaruli T. for the ANTARES Collaboration, Int. J. Mod. Phys., A24, 1656 (2009);

[22] Abe K. et al., Astrop. J., 652, 198 (2006);

[23] Migneco E. et al., Nucl. Instr. Meth., A588, 111 (2008);

[24] Aiello S. et al., Astrop. Phys, 33, 263 (2010);

[25] Technical Design Report for a Deep-Sea Research Infrastructure Incorporating a Very Large Volume Neutrino Telescope in the Mediterranean Sea (2010). In preparation;

[26] Aharens et al., Astropart. Phys., 20, 507 (2004)

[27] Askaryan G. A., JETP, 14, 441 (1962);

[28] Saltzberg D. et al., Phys. Rev. Lett., 86, 2802 (2001);

[29] Barwick S.W. et al., Phys. Rev. Lett., 96, 171101 (2006);

[30] Riccobene G., Journal of Physics: Conference Series, 136, 022053 (2008);

[31] Askaryan G. A., Atom. Ener., 3, 153 (1957);

[32] Learned J. G., Phys. Rev., D19, 3293 (1979);

[33] Urick R. J., Sound Propagation in the Sea, Peninsula Publishing, (1982);

[34] Descamps F. et al. al., Nucl. Instr. Meth., A604-1S, S175 (2009);

[35] Lahman R. et al., Nucl. Instr. Meth., A604-1S, S158 (2009);

[36] Riccobene G. et al., Nucl. Instr. Meth.,A604-1S, S149 (2009);

[37] Sapienza P. and Riccobene G., Riv. Nuovo Cimento, 32, 591 (2009) 